\title{
Lithium-ion battery models: a comparative study and a model-based powerline communication
}

\author{
Fida Saidani $^{1}$, Franz X. Hutter ${ }^{1}$, Rares-George Scurtu ${ }^{2}$, Wolfgang Braunwarth ${ }^{2}$, and Joachim N. Burghartz ${ }^{1}$ \\ ${ }^{1}$ Institut für Mikroelektronik Stuttgart (IMS CHIPS), Allmandring 30a, 70569 Stuttgart, Germany \\ ${ }^{2}$ Zentrum für Sonnenenergie- und Wasserstoff-Forschung Baden-Württemberg (ZSW), Lise-Meitner-Straße 24, \\ 89081 Ulm, Germany
}

Correspondence to: Fida Saidani (saidani@ims-chips.de)

Received: 19 December 2016 - Revised: 13 March 2017 - Accepted: 18 March 2017 - Published: 21 September 2017

\begin{abstract}
In this work, various Lithium-ion (Li-ion) battery models are evaluated according to their accuracy, complexity and physical interpretability. An initial classification into physical, empirical and abstract models is introduced. Also known as "white", "black" and "grey" boxes, respectively, the nature and characteristics of these model types are compared. Since the Li-ion battery cell is a thermo-electrochemical system, the models are either in the thermal or in the electrochemical state-space. Physical models attempt to capture key features of the physical process inside the cell. Empirical models describe the system with empirical parameters offering poor analytical, whereas abstract models provide an alternative representation.

In addition, a model selection guideline is proposed based on applications and design requirements. A complex model with a detailed analytical insight is of use for battery designers but impractical for real-time applications and in situ diagnosis. In automotive applications, an abstract model reproducing the battery behavior in an equivalent but more practical form, mainly as an equivalent circuit diagram, is recommended for the purpose of battery management. As a general rule, a trade-off should be reached between the high fidelity and the computational feasibility. Especially if the model is embedded in a real-time monitoring unit such as a microprocessor or a FPGA, the calculation time and memory requirements rise dramatically with a higher number of parameters.

Moreover, examples of equivalent circuit models of Lithium-ion batteries are covered. Equivalent circuit topologies are introduced and compared according to the previously introduced criteria. An experimental sequence to model a $20 \mathrm{Ah}$ cell is presented and the results are used for the purposes of powerline communication.
\end{abstract}

\section{Introduction}

Modern electronics come with significant energy consumption costs. Features like colorful displays, powerful processors and wireless communication are energy-hungry (Rao et al., 2003; Hu et al., 2012). Unfortunately, battery technology has not kept up with the consumer electronics growth, which has made the battery the bottleneck component in portable and automotive electronics. Among all different battery technologies, Lithium-ion (Li-ion) batteries dominate the consumer market. In 2013, five billion Li-ion cells were sold worldwide; modern technologies store twice as much energy per weight as the first commercial versions introduced by Sony in 1991 (Van Noorden, 2014).

As shown in Fig. 1, the high energy density and compactness of Li-ion batteries make them the first choice for energy storage in laptops, cameras, mobile phones, and other applications (Van Noorden, 2014).

However, their reliability and lifetime are limited and depend considerably on environmental conditions and history of usage. Therefore, accurate and effective battery models are needed for monitoring purposes (Hu et al., 2012; Rao et al., 2003). Being also used in electric and hybrid vehicles, higher requirements on battery design and management systems are set to ensure a safe, reliable and cost-efficient operation. Therefore, accurate and computationally feasible battery models are important. This paper presents a comparative study of various Li-ion battery cells, according to welldefined criteria.

Section 2 presents an insightful view into the structure of a Li-ion cell and introduces the comparison criteria. 


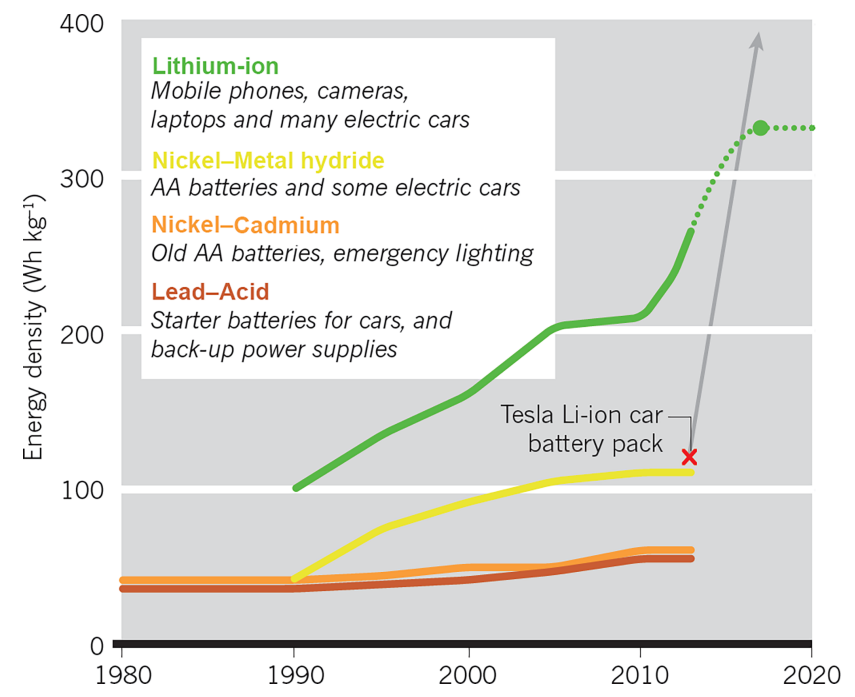

Figure 1. Comparison of energy densities for different battery technologies

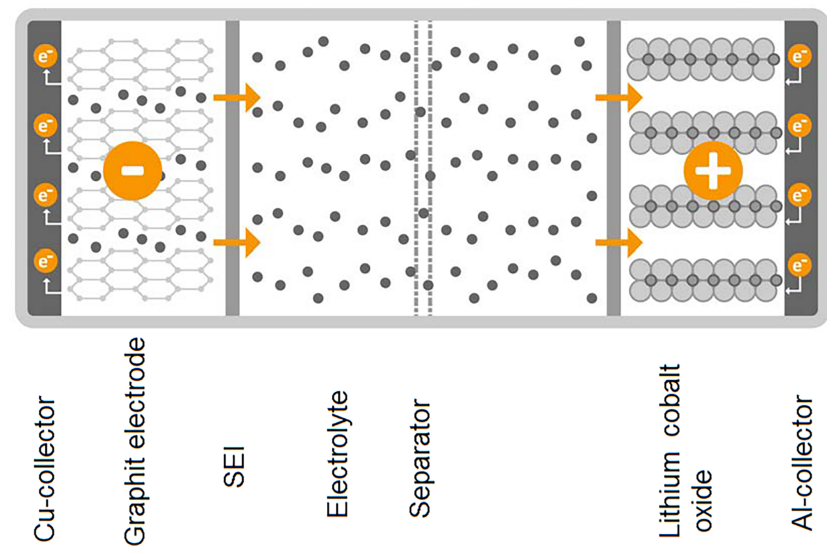

Figure 2. The structure of a Li-ion cell

Section 3 introduces in detail the different battery models widely used in the literature and concludes with a comparative overview and an evaluation in the context of automotive applications.

Section 4 reports on the experimental modeling of a $20 \mathrm{Ah}$ Li-ion battery hardcase cell and on an attempt to realize a model-based powerline communication.

\section{Modeling of Lithium-ion batteries: a guide}

The battery is a thermo-electro-chemical system. In this work, models in the electrochemical domain are of interest (Schmidt, 2013). Figure 2 (Rahimzei et al., 2012) shows the composition of a Li-ion battery cell.

Every cell necessarily has 4 components: 2 electrodes, a separator and an electrolyte. The designations of the electrodes as anode and cathode are relative and are used alter- nately, depending on whether the cell is in the charging or discharging cycle. In both cases, a reduction-oxidation (redox) reaction is responsible for the current flow. In the following, we will use the designations relative to the discharge cycle. That means that the anode is the electrically negative electrode delivering electrons to the outside circuit, and the cathode is the electrically positive electrode.

The electrolyte is generally a lithium salt (e.g. Lithium hexafluorophosphate, $\mathrm{LiPF}_{6}$ ) dissolved in an organic solvent (Worthman, 2015). The separator is a porous membrane (typically Polypropylene or Polyethylene) separating the anode and cathode spatially and electrically. It is ion-permeable so that only $\mathrm{Li}^{+}$ions (and not the electrons) can migrate inside the cell between electrodes (von Srbik et al., 2016).

If a load is connected to the cell, the cell delivers current to the outside circuit, i.e. the cell is being discharged. An oxidation reaction is happening at the anode (generally carbon, i.e. graphit, hosting Li atoms), according to Reaction (1).

$x \cdot \mathrm{LiC}_{6} \rightarrow x \cdot \mathrm{Li}^{+}+x \cdot e^{-}+x \cdot \mathrm{C}_{6}$

The cathode (typically a Lithium Cobalt oxide) hosts a reduction reaction according to Reaction (2).

$\mathrm{Li}_{1-x} \mathrm{CoO}_{2}+x \cdot \mathrm{Li}^{+}+x \cdot e^{-} \rightarrow \mathrm{LiCoO}_{2}$

The $x \mathrm{Li}^{+}$ions liberated in Reaction (R1) at the anode migrate in the electrolyte through the separator to the cathode and accumulate there. The electrons liberated in the same reaction flow into the outside circuit through the load and accumulate likewise in the cathode (Worthman, 2015). This reaction continues until the concentration of active species comes under a certain threshold and can no longer be sustained. Temperature and other environmental conditions, as well as discharge rates affect the speed with which this voltage cut off is reached. The discharge cycle is then followed by a charging cycle where Reactions (R1) and (R2) take place in the opposite direction.

Numerous models for Li-ion cells and batteries are available in the literature. Modeling of the battery is important during the design as well as the run time stage. During the design stage, models help battery technology specialists develop better and more robust battery systems with less costs. During the run time stage, accurate models help deliver information about the battery's parameters of interest under given system constraints. Possible applications of models are the optimization of battery management, i.a. cell scheduling algorithms and the shaping of the discharge profile of the battery under performance constraints. This enables the maximization of the battery life time by trading off performance using information about the State of Charge (SoC).

This comparative study attempts to compare models widely used in the literature according to well-defined criteria. The models will then be evaluated in the particular context of Hybrid Electric Vehicles (HEV). The models available in the literature are classified into physical, empirical and abstract models. Their comparison is based on three criteria: 


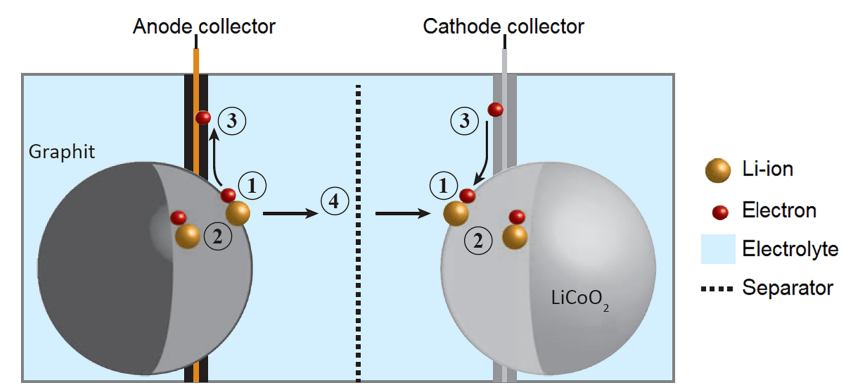

Figure 3. Physical processes taking place into a Li-ion cell

the accuracy of the model, the complexity of the model, and the physical interpretability offered by the model (Rao et al., 2003).

The accuracy defines how closely the model can predict battery state parameters and/or values of the battery variables of interest. The complexity sets the number of parameters needed for the model. Depending on the complexity level, computations or in situ estimations will take a more or less considerable time, which would put into question the model suitability for real-time applications. Also, complex models come with configuration effort costs. The physical interpretability can also be defined as the analytical insight the model provides. A model with a high level of physical interpretability offers a good analytical understanding of the physics inside the battery. The usefulness of this insight depends on the application.

\section{Li-ion battery cell models: an overview}

\subsection{Physical models}

Also known as white boxes (Schmidt, 2013), physical models are low-level models with a high accuracy level (Rao et al., 2003). They describe the structure of the materials and depict the complex electrochemical phenomena happening inside the cell, namely thermodynamics, active species kinetics and transport phenomena.

Schmidt (2013) presented an overview of the physical phenomena as shown in Fig. 3. Four main processes are taking place in three regions of operation: the two solid phases of the electrodes material, and the liquid phase of the electrolyte.

The first process (process (1) in Fig. 3) is the passing through of charges and takes place in the first region of operation: the solid phase of the electrode. This process describes the de- or intercalation of the Lithium ions into the active material. The second process (process (2) in Fig. 3), taking place in the same region, is the solid state diffusion of Lithium ions compelled by the concentration gradient of ions between the surface and the bulk of the electrode. The third process (process (3) in Fig. 3) designates the electrons conduction from one electrode to another through the outside circuit. The fourth process (process (4) in Fig. 3) is the ions conduction in the electrolyte through the separator. It is also forced by the concentration gradient and is based on diffusion but happens at a higher rate than in solids.

Based on interdependent differential equations, a large number of parameters is needed along with a high configuration effort to establish a physical model. Diffusion coefficients as well other material related characteristics are important parameters in the equations (Keil and Jossen, 2012). Examples of research attempting on physical modeling of Liion batteries are presented by Smith et al. (2007) and Verbrugge et al. (2000). Such models offer a high analytical insight, which might be interesting for battery technology specialists and material scientists. However, from an electrical engineering point of view, such an insight is not necessary. With sometimes more than 50 parameters, the complexity is significantly high. This increases the requirements for needed memory and computation time. Some models were reported to have run into over-fitting problems ( $\mathrm{Hu}$ et al., 2012).

\subsection{Empirical models}

Empirical models are also called black boxes (Schmidt, 2013) because of the poor insight they offer into the system. They are based on empirical parameters which do not have any physical significance in some cases (Rao et al., 2003). The mathematical approaches used to define the transfer function from the inputs to the outputs of the black box make these models easy to configure, and able to deliver quick responses and predictions. However, their accuracy remains limited, especially if the model is too simple (Rao et al., 2003). The accuracy and the physical insight can be improved if combined with a low-level model (Sect. 3.1).

More sophisticated models achieve better results based on stochastic models and/or fuzzy logic and can deliver battery related parameters. Examples for such models are presented by Yu (2015) and Wu et al. (2012). The work of Jiani et al. (2014) is also based on a similar mathematical approach; the model presented itself is rather to be classified under the category of the abstract models, which is presented in Sect. 3.3.

\subsection{Abstract models}

Also known as grey boxes (Schmidt, 2013), abstract models provide an alternative representation of the physical entity. In the context of Li-ion batteries, these models present a different, but equivalent representation of the battery system. Different forms are therefore possible; the representation as an electrical equivalent circuit is, however, very popular. Examples are provided by Hu et al. (2012), Rahmoun and Biechl (2012), Huria et al. (2012) and Ouannes et al. (2014). The circuit-based models are simple and practical because they allow for the complex electrochemical process to be replaced by a simple electrical circuit. The correlation with battery dy- 


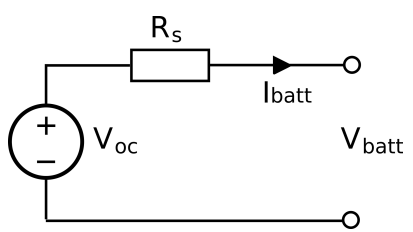

Figure 4. The zero-time-constant model of a Li-ion cell

namics is therefore preserved, without compromising much of the accuracy (Moss et al., 2008).

The configuration cost needed for these models is reduced compared to low-level models, but look-up tables are still required in order to match experimental data. The complexity is flexible depending on the computation unit and memory resources. More complex but also more accurate models take second-order effects like temperature, aging and capacity fading into consideration, as presented by Huria et al. (2012) and Erdinc et al. (2009).

\subsubsection{Simple circuits as Li-ion battery models}

The simplest form of an equivalent circuit model to represent a battery is a zero-time-constant circuit as shown in Fig. 4. If the user is not interested in battery dynamics, this model is able to represent the static behavior of the system. The Open Circuit Voltage (OCV) is directly related to the State of Charge (SoC) (Keil and Jossen, 2012), as defined in Eq. (3),

$\mathrm{SoC}=\frac{C_{\text {current }}}{C_{\text {full }}} \cdot 100 \%$,

wherein $C_{\text {current }}$ is the charge amount currently available in the cell and $C_{\text {full }}$ is the capacity of the same cell when fully charged (Schmidt, 2013). The model equation is expressed in Eq. (4):

$V_{\text {batt }}=V_{\mathrm{OC}}-R_{\mathrm{S}} \cdot I_{\text {batt }}$.

The curve of the OCV as a function of time shows a constantly decreasing voltage for given discharge conditions. The important parameters affecting the discharge process are the discharge current, the temperature and the charging/discharging history (Rao et al., 2003).

For similar charging conditions, higher discharge currents induce higher discharge rates and a higher decreasing rate of the OCV towards the cut off threshold (Keil and Jossen, 2012).

The temperature effect on the discharge behavior is seen at temperatures much below room temperature, where the chemical activity decreases and the internal resistance of the battery consequently increases. At temperatures much higher than room temperature, the internal resistance decreases. This enhances a higher rate of chemical activity, therefore however inducing a self-discharge effect (Rao et al., 2003).

The history effect is difficult to characterize. Effects like capacity fading, charge recovery, electrolyte decomposition and material dissolution come into play (Rao et al., 2003).

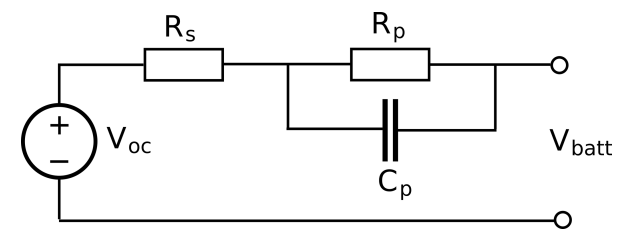

Figure 5. The one-time-constant model of a Li-ion cell

The main disadvantage of zero-time-constant models is that they do not account for cell dynamics. The one-timeconstant models overcome this drawback by adding one $\mathrm{RC}$ element in series to the resistance (Fig. 5), which describes the transient response of the battery during charging/discharging.

Adding more RC elements improves the accuracy of the model and its consideration of the dynamic behavior (Sect. 3.3.2) but increases simultaneously its complexity (Huria et al., 2012; Moss et al., 2008).

$\mathrm{Hu}$ et al. (2012) present a comparative overview of circuit models, with no or up to $3 \mathrm{RC}$-elements with consideration of self-correction and hysteresis effect (the discrepancy between the charging and discharging process).

\subsubsection{Models based on the impedance spectrum}

In order to depict the cell dynamics at middle and higher frequencies, the method of the electrochemical impedance spectroscopy (EIS) is applied. A linear time invariant system is required for the application of EIS measurements. Since the batteries are highly nonlinear during their charging and discharging cycles, the EIS is applied at given SOC values where the battery has had a compulsory rest of a given period, so that linearity can be sufficiently guaranteed (Keil and Jossen, 2012).

The impedance $Z$ is a complex variable represented in the Nyquist plot as real $(\Re(Z)=Z \prime)$ and imaginary $(\Im(Z)=$ $Z$ II) parts. Figure 6 (Keil and Jossen, 2012) shows a qualitatively typical Nyquist curve resulting from the EIS measurements of a Li-ion battery. Quantitatively, the values depend on different factors, i.e. the temperature, the SoC and the load current.

The curve can be subdivided according to the frequency ranges into different sections, which can be associated with well-defined physical and electrochemical phenomena taking place inside the cell.

The section with $Z$ II $<0$ consists of three more or less well recognizable arcs. The arc at very low frequencies (near DC, arc (i) in Fig. 6) is associated with the diffusion behavior as introduced in Sect. 3.1 and is represented by the Solid-State Warburg Impedance (Moss et al., 2008; Keil and Jossen, 2012). The second arc at slightly higher frequencies (arc (ii) in Fig. 6) corresponds to the charge transfer kinetics. The third and smallest arc near the real axis (arc (iii) in 
Table 1. Li-ion battery models comparison

\begin{tabular}{lllll}
\hline Models & Accuracy & Complexity & Physical interpretability & Suited application \\
\hline Physical & Very high & $\begin{array}{l}\text { High } \\
(>50 \text { parameters })\end{array}$ & High & Battery system design stage \\
& +++ & - & ++ & \\
\hline Empirical & Medium & Low & Low & Predictions of life time and efficiency \\
& & $(2-3$ parameters $)$ & - & \\
\hline Abstract & Medium & $\begin{array}{l}\text { Medium to low } \\
\text { (from 2 up to 30) }\end{array}$ & Limited to acceptable & Real-time monitoring and diagnosis \\
& & + & + & \\
\hline
\end{tabular}

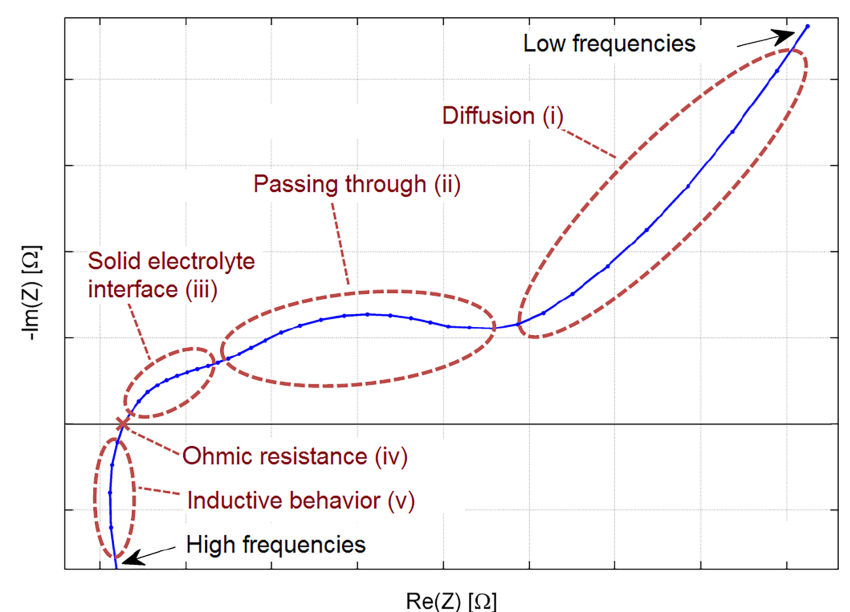

Figure 6. Typical EIS response represented as a Nyquist Plot.

Fig. 6) represents the interlayer effects at the solid electrolyte interface (Keil and Jossen, 2012; Moss et al., 2008).

The intersection with the horizontal axis $\left(Z_{\prime \prime}^{\prime \prime}=0\right.$, point (iv) in Fig. 6) represents the total ohmic resistance of the system, including the electrolyte resistance, the contact resistance and the electronic contacts. This point generally occurs at a frequency in the $\mathrm{KHz}$ range but can vary considerably with the cell design and the used materials (Keil and Jossen, 2012).

The highest frequencies show an inductive behavior with $Z_{\prime \prime}>0$ (tail (v) in Fig. 6) corresponding to the electrode porous structure and to the battery leads.

The equivalent circuit modeling of the impedance response is introduced by Moss et al. (2008) as in Fig. 7, wherein $C_{\mathrm{dl}}$ is the electrochemical double layer capacitance, $R_{\mathrm{CT}}$ is the faradaic charge transfer resistance, $C_{\mathrm{int}}$ is the intercalation capacitance corresponding to the accumulation of the Lithium ions within the electrode matrix, and $Z(\omega)$ is the Warburg solid-state impedance.

Moss et al. (2008) suggest the substitution of the Warburg diffusion impedance with a chain of RC elements. The new

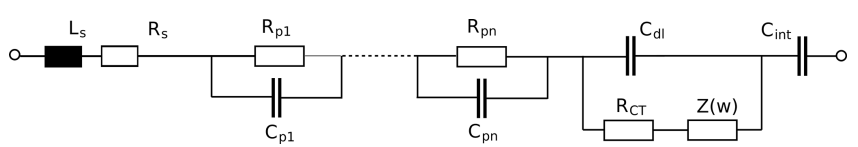

Figure 7. A complex equivalent circuit to model the EIS response.

chain does not mirror the impedance of the Warburg element but represents an approximation with an acceptable accuracy. Taking this approximation technique into consideration, the pertinence of the general assumption widely used in the literature of modeling Li-ion battery cell as a voltage source in series with a resistance $R_{\mathrm{S}}$ and a chain of parallel RC elements is verified. This is only possible thanks to KramersKronig relations (Schmidt, 2013), which can be applied on equivalent networks, and which suggest that many different circuits can have the same or similar impedance responses without having the same topology. In other words, the battery can be modeled using an easier topology with circuit elements which do not have any direct physical interpretation. This model is valid, because it has the same frequency response, and would be easier to parametrize.

The choice of the number of $\mathrm{RC}$ elements results in a trade-off between fidelity and complexity (Huria et al., 2012; Moss et al., 2008; Keil and Jossen, 2012). The model can be extended with a parasitic branch, which is disregarded for cells with high coulombic efficiencies (Huria et al., 2012), and with a look-up table showing the temperature dependency of the circuit elements' values.

\subsection{Models comparison and evaluation for hybrid electric vehicle applications}

Table 1 presents a comparative overview of the introduced models according to the comparison criteria previously explained. As a conclusion, a trade-off between accuracy and model complexity should be accepted. Simpler models are less expensive and less susceptible to uncertainties. Complex models need more time to solve algorithms for State of Charge (SoC) and State of Health $(\mathrm{SoH})$ estimations. De- 


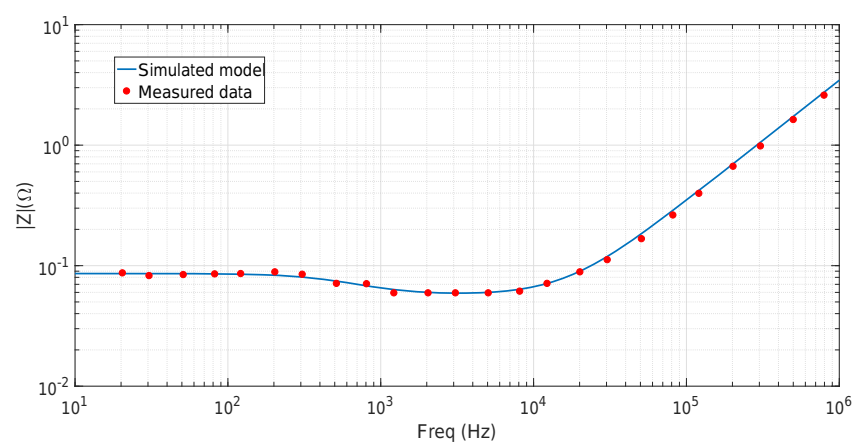

Figure 8. Coarse impedance measurements of a $20 \mathrm{Ah}$ hardcell cell.

pending on the application, the model extraction method and usefulness can be evaluated. In a laboratory environment, more time can be invested in order to extract a more accurate model. On the field, quicker results are needed to evaluate whether the cells are i.e. still suitable for their first life application (Li et al., 2014). In automotive applications, Li-ion cells modeling is used for the purposes of battery management system (BMS) operation. In the following the criteria are adapted to the requirements on BMS for HEV applications.

Thorough understanding of cell chemistry is not needed, although beneficial. The model is embedded into a real-time monitoring unit, such as a microcontroller or an FPGA. Simplicity is therefore required. In fact, the calculation time and memory requirements rise dramatically with higher complexity levels, so the computational feasibility compromises the accuracy of the model. Abstract models are therefore the most suitable for this application.

\section{Application: a powerline communication concept using an experimentally extracted model}

\subsection{Impedance measurements of a $20 \mathrm{Ah}$ hardcase cell}

This subsection deals with the experiment of modeling a Li-ion cell of a typical capacity of $20 \mathrm{Ah}$. The first experiment was done using an LCR meter at the frequency values shown in Fig. 8. This measurement setup included parasitics which compromised the model accuracy. A more accurate model can be obtained from impedance spectroscopy measurements.

The following experimental sequence consists in Electrochemical Impedance Spectroscopy (EIS) measurements between $10 \mathrm{mHz}$ and $2.5 \mathrm{KHz}$ at open circuit voltage (OCV) values, with $V_{\mathrm{ac}}=2.5 \mathrm{mV}$.

Using Galvanostatic Cycling with Potential Limitation (GCPL), the method of Constant Current Constant Voltage (CCCV) is applied at the cell connectors. For charging, a constant voltage of $4.1 \mathrm{~V}$ is applied for $1 \mathrm{~h}$ or until the current $I<C / 20$. In this and similar contexts, the current intensity

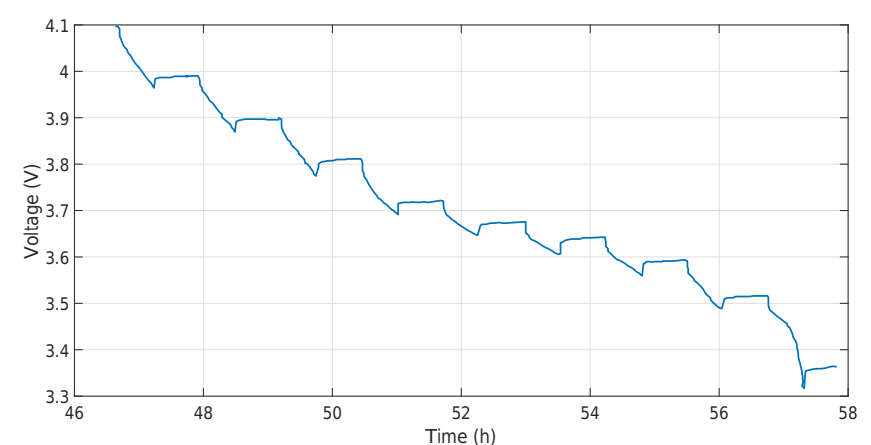

Figure 9. OCV values for EIS Spectra.

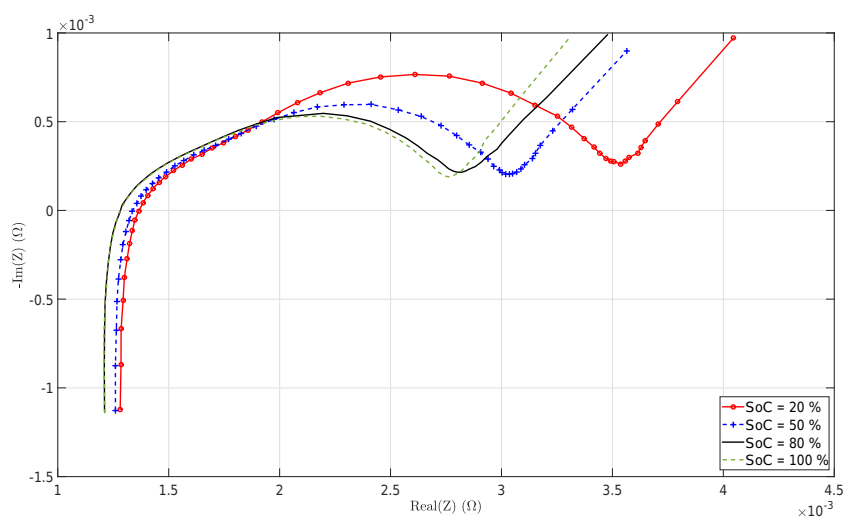

Figure 10. EIS recorded spectra for a 20 Ah battery cell.

is given in $C$-ratios. One $C$-ratio is defined as the current intensity obtained by dividing the rated capacity by $1 \mathrm{~h}$, given in this case by Eq. (5) (Keil and Jossen, 2012). For discharging, a constant current of $0.2 C$ is applied until a voltage of $3 \mathrm{~V}$ is reached.

$1 C=20 \mathrm{Ah} / 1 \mathrm{~h}=20 \mathrm{~A}$

The GCPL discharge is applied with $0.2 C$ in 9 steps. After each step an EIS measurement is carried out after a rest period of $30 \mathrm{~min}$, as shown in Fig. 9.

Figure 10 shows the EIS recorded spectra at several SoC values.

The analysis carried out considers one defined set of temperature and environmental conditions for the experimental sequence and does not account for different rest periods and/or different charge and discharge currents. In addition, effects such as the hysteresis, the temperature and aging factors, as well as mechanical pressure are not considered.

The same data of Fig. 10 is represented in Figs. 11, 12 and 13 as the recorded magnitudes and phases as a function of the frequency. Figure 12 represents a zoom into the low frequency spectrum of the data $(\mathrm{DC}-50 \mathrm{~Hz})$. The phase continues however to increase in the positive direction up to $2.5 \mathrm{KHz}$ as shown in Fig. 13. 


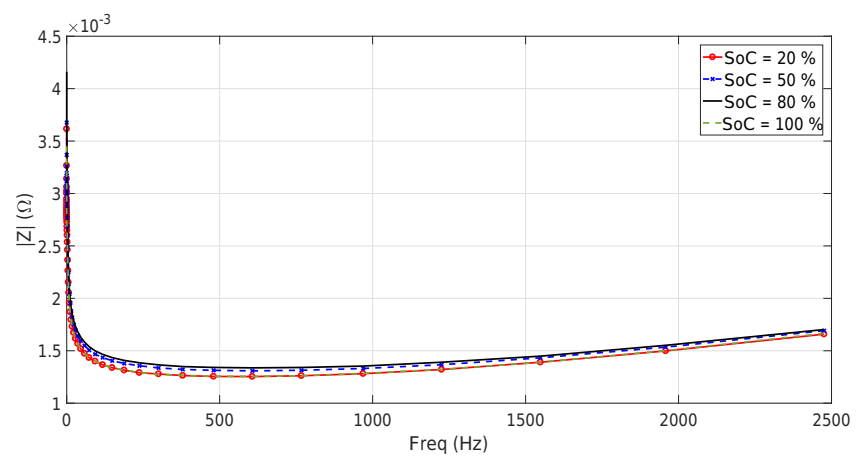

Figure 11. Recorded magnitudes for a $20 \mathrm{Ah}$ battery cell.

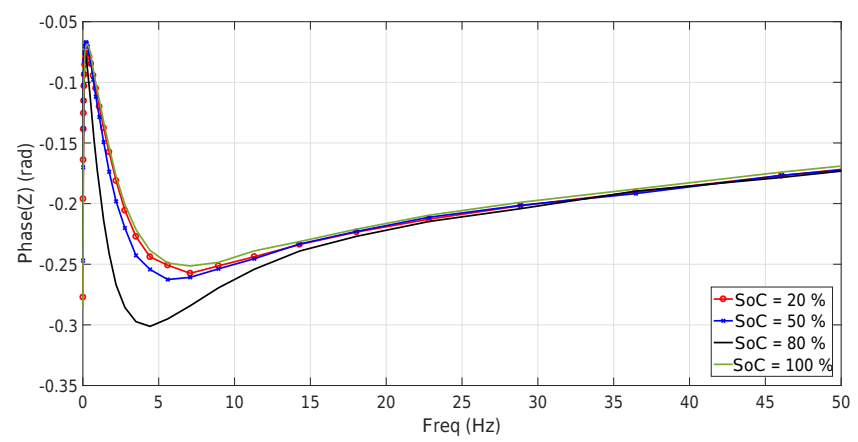

Figure 12. Recorded phases for a $20 \mathrm{Ah}$ battery cell up to $50 \mathrm{~Hz}$.

\subsection{Experimental modeling of a $20 \mathrm{Ah}$ hardcase cell}

Figure 10 shows that the EIS recorded data of the $20 \mathrm{Ah}$ hardcase cell follow the qualitative pattern introduced in Sect. 3.3.2. The cell will be modeled for $\mathrm{SoC}=80 \%$ using an equivalent circuit. In the following, the circuit topology and elements will be discussed.

Figures 11, 12 and 13 show an inductive tail at the highest frequencies, since the magnitude goes moderately higher with higher frequencies, along with a positive phase. We will use a model consisting of one resistance $R_{\mathrm{S}}$, representing the internal resistance of the battery, and one inductance $L_{\mathrm{s}}$ in series. Also, in order to depict the dynamic behavior of the battery, 2 RC elements will be used as explained in Sect. 3.3.2. The complete circuit is shown in Fig. 14. The total impedance of the circuit $Z_{\text {batt }}$ is given by Eq. (6).

$$
Z=R_{\mathrm{s}}+j \omega L_{\mathrm{s}}+\frac{R_{\mathrm{p} 1}}{1+j \omega R_{\mathrm{p} 1} C_{\mathrm{p} 1}}+\frac{R_{\mathrm{p} 2}}{1+j \omega R_{\mathrm{p} 2} C_{\mathrm{p} 2}}
$$

The modeling sequence consists in finding the values for the circuit elements of Fig. 14 and simulating the circuit frequency response using the circuit simulation software LtSpice (LTspice, 1998-2017). A sweep of the different parameters is carried out in order to find the best fitting between simulated and measurement data.

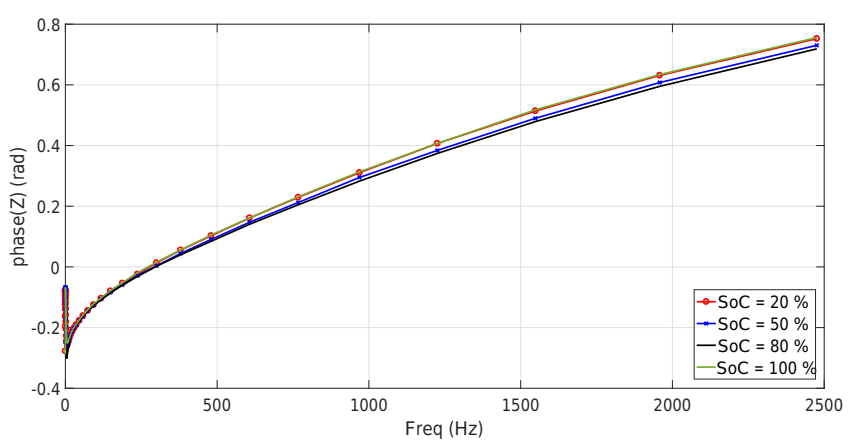

Figure 13. Recorded phases for a 20 Ah battery cell.

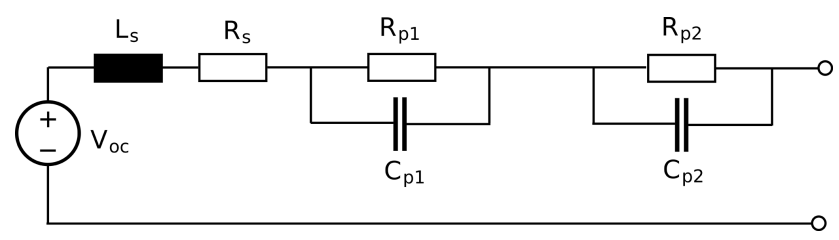

Figure 14. The model used to model the $20 \mathrm{Ah}$ cell.

In order to simplify the modeling experiment, it was assumed that $R_{\mathrm{S}}$ is constant throughout the different $\mathrm{SoC}$ and that only $R_{\mathrm{p}, \mathrm{i}}$ and $C_{\mathrm{p}, \mathrm{i}}$ values are a function of the battery dynamics. Table 2 shows the values of the parameters that deliver the best fitting between the simulated and measured data for $\mathrm{SoC}=80 \%$. Figure 15 shows the comparison of measured and simulated impedance data for this case.

\subsection{A powerline communication concept}

The goal behind the modeling of the $20 \mathrm{Ah}$ battery cell and the characterization of its frequency response was to realize a new communication concept using the battery impedance. We are interested in monitoring the battery operation by using integrated sensors and communicating the output of these sensors to an external monitoring unit.

The operating principle of the communication scheme is similar to the well-known powerline communication (Ouannes et al., 2014). However, the signal is not carried over to the powerline using a capacitive or an inductive element. In this case, we connect an AC current source $I_{\mathrm{AC}}$ in parallel to the battery terminals as shown in Fig. 16. During the monitoring phase, the cells in series constituting the battery module will be disconnected from the load. The current will then flow directly back into the cell.

Using the battery impedance $Z_{\text {batt }}$ as described in Sect. 4.2, the high frequency current $I_{\mathrm{AC}}$ will induce a high frequency voltage $V_{\mathrm{AC}}=I_{\mathrm{AC}} \cdot Z_{\mathrm{batt}}$ above the DC level of the battery. This AC voltage can consequently be used as the carrier wave to modulate binary data using a conventional modulating scheme such as phase-shift keying (Ouannes et al., 2014). The signal can then be tapped at the end of the chain and demodulated using appropriate circuitry. 


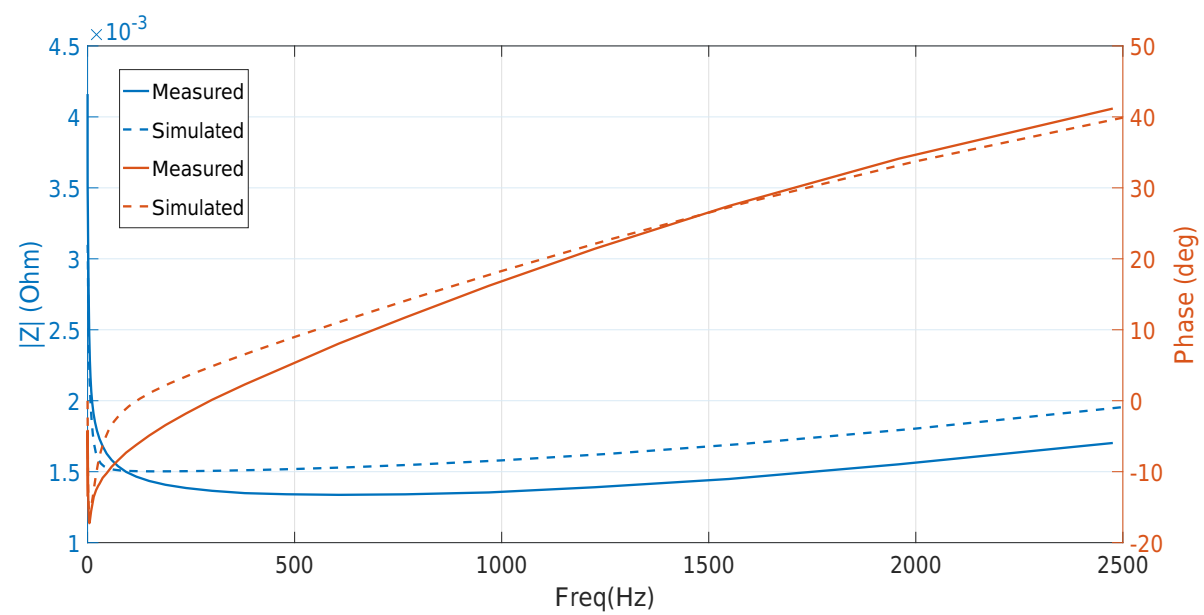

Figure 15. Measured and simulated impedance at $\mathrm{SoC}=80 \%$.

Table 2. Model elements values for $\mathrm{SOC}=80 \%$.

\begin{tabular}{lr}
\hline Parameters & Values \\
\hline$R_{\mathrm{S}}$ & $0.5 \mathrm{~m} \Omega$ \\
$L_{\mathrm{S}}$ & $80 \mathrm{nH}$ \\
$R_{\mathrm{p} 1}$ & $1 \mathrm{~m} \Omega$ \\
$C_{\mathrm{p} 1}$ & $80 \mathrm{~F}$ \\
$R_{\mathrm{p} 2}$ & $0.6 \mathrm{~m} \Omega$ \\
$C_{\mathrm{p} 2}$ & $50 \mathrm{~F}$ \\
\hline
\end{tabular}

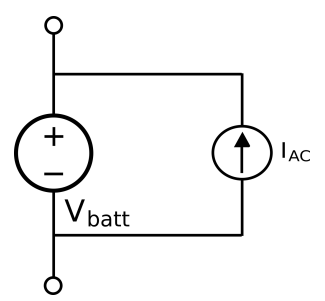

Figure 16. The powerline communication concept.

Figure 17 shows the resulting $\mathrm{AC}$ voltage when an input sine wave of $V_{\mathrm{pp}}=4 \mathrm{~V}$ and $f=1 \mathrm{MHz}$ is applied. At lower frequencies, no AC signal was detected. For frequencies in the $\mathrm{MHz}$ range, and as seen in this example, the $\mathrm{AC}$ voltage output is very low (constantly under $10 \mathrm{mV}$ ). The possible explanation is that the battery impedance $Z_{\text {batt }}$ is in fact too low to support the signal.

Since it was not developed for communiation purposes, the powerline is a harsh environment in terms of noise figures and frequency selectivity, especially at interesting frequency bands for the communication (Ouannes et al., 2014). Consequently, the new concept communication cannot be realized. The desired signal is limited by the low internal battery impedance.

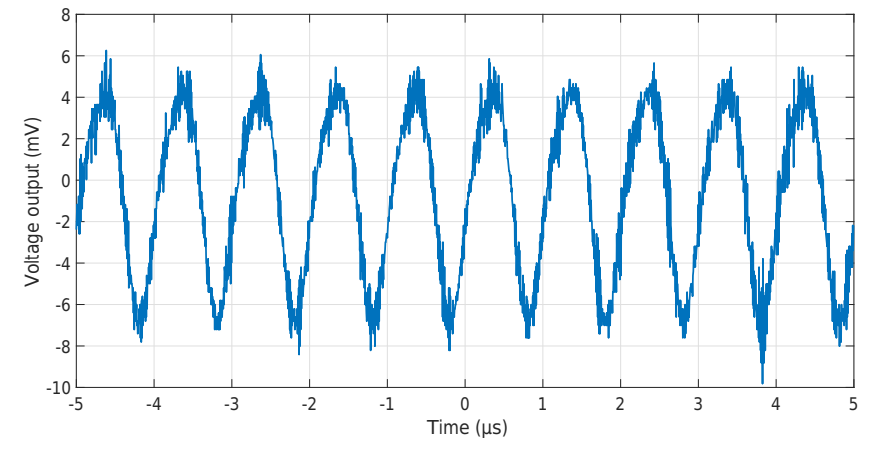

Figure 17. AC signal output at the battery cell.

\section{Conclusions}

This work presents a comparative overview of Li-ion battery models used in the literature. An explanation of the model types as well as a guide into the selection of the best suited model depending on the application are presented.

In the context of electrical engineering and for the special purpose of battery management and monitoring, abstract models taking the form of equivalent circuits are a popular and valid choice.

Also, a trade-off between the complexity of the equivalent circuit (mainly the number of RC elements) and its accuracy should be accepted. More RC elements help depict the battery dynamics more accurately but increase the computation and memory requirements of the model, which compromises its suitability for real-time applications.

An experiment of modeling a $20 \mathrm{Ah}$ as an equivalent electronic circuit is presented. Impedance spectroscopy measurements were carried out, a circuit topology was chosen, and a fitting of the circuit parameters was done to match simulated and measured data. 
Finally, a new powerline communication concept is introduced. The new concept requires the availability of an impedance with a sufficiently high inductive component. However, the total impedance of the battery was found to be too low to deliver the desired AC signal.

Data availability. The data used in this paper, unless cited or otherwise referred to, are measurements/simulations conducted in laboratory environments in one the institutes listed in the affiliations of the authors. This data is not available online.

Competing interests. The authors declare that they have no conflict of interest.

Acknowledgements. This work has been conducted within the scope of the project Smart-LiB: Intelligente Li-Ionen-Batterien in der Fertigung und Anwendung. The authors gratefully acknowledge the financial support of Ministerium für Finanzen Baden-Württemberg (MFW-BW). The authors would also like to thank T. Zaki for setting the first bricks of the modeling experiments and M. Nawito for helping with the analysis of the EIS data.

Edited by: J. Anders

Reviewed by: two anonymous referees

\section{References}

Erdinc, O., Vural, B., and Uzunoglu, M.: A dynamic lithium-ion battery model considering the effects of temperature and capacity fading, in: 2009 International Conference on Clean Electrical Power, 383-386, 2009.

$\mathrm{Hu}, \mathrm{X} ., \mathrm{Li}, \mathrm{S}$., and Peng, H.: A comparative study of equivalent circuit models for Li-ion batteries, J. Power Sources, 198, 359367, 2012.

Huria, T., Ceraolo, M., Gazzarri, J., and Jackey, R.: High fidelity electrical model with thermal dependence for characterization and simulation of high power lithium battery cells, in: 2012 IEEE International Electric Vehicle Conference, 1-8, 2012.

Jiani, D., Zhitao, L., Youyi, W., and Changyun, W.: A fuzzy logicbased model for Li-ion battery with SOC and temperature effect, in: 11th IEEE International Conference on Control Automation (ICCA), 1333-1338, 2014.

Keil, P. and Jossen, A.: Aufbau und Parametrierung von Batteriemodellen, in: 19. DESIGN und ELEKTRONIKEntwicklerforum, Batterien und Ladekonzepte, 2012.
Li, X., Wang, T., Pei, L., Zhu, C., and Xu, B.: A comparative study of sorting methods for Lithium-ion batteries, in: 2014 IEEE Conference and Expo Transportation Electrification Asia-Pacific (ITEC Asia-Pacific), 1-6, 2014.

LTspice: XVII, Linear Technology, US Pacific, 1998-2017.

Moss, P. L., Au, G., Plichta, E. J., and Zheng, J. P.: An Electrical Circuit for Modeling the Dynamic Response of Li-Ion Polymer Batteries, Journal of Electrochemical Society 155 , Nr. 12, A986-A994, 2008.

Ouannes, I., Nickel, P., and Dostert, K.: Cell-wise monitoring of Lithium-ion batteries for automotive traction applications by using power line communication: battery modeling and channel characterization, in: 18th IEEE International Symposium on Power Line Communications and Its Applications, 24-29, 2014.

Rahimzei, E., Sann, K., and Moritz, V.: Kompendium: Li-IonenBatterien, Grundlagen, Bewertungskriterien, Gesetze und Normen, im BMWi Förderprogramm IKT für Elektromobilität II: Smart Car - Smart Grid - Smart Traffic, 2012.

Rahmoun, A. and Biechl, H.: Modelling of Li-ion batteries using equivalent circuit diagrams, Przeglad Elektrotechniczny, 88, 152-156, 2012.

Rao, R., Vrudhula, S., and Rakhmatov, D. N.: Battery Modeling for Energy-Aware System Design, Computer, 36, 77-87, 2003.

Schmidt, J. P.: Verfahren zur Charakterisierung und Modellierung von Lithium-Ionen Zellen, vol. 25 of Schriften des Instituts für Werkstoffe der Elektrotechnik, Karlsruher Institut für Technologie, KIT Scientific Publishing, Karlsruhe, 2013.

Smith, K. A., Rahn, C. D., and Wang, C.-Y.: Control oriented 1D electrochemical model of lithium ion battery, Energ. Convers. Manage., 48, 2565-2578, 2007.

Van Noorden, R.: The rechargeable revolution: A better battery, Nature, 507, 26-28, 2014

Verbrugge, M. W., Koch, B. J., and Schneider, E. W.: Mass Transport in Lithium-battery Solvents, J. Appl. Electrochem., 30, 269 275, https://doi.org/10.1023/A:1003984511421, 2000.

von Srbik, M.-T., Marinescu, M., Martinez-Botas, R. F., and Offera, G. J.: A physically meaningful equivalent circuit network model of a lithium-ion battery accounting for local electrochemical and thermal behaviour, variable double layer capacitance and degradation, 325, 171-184, J. Power Sources, https://doi.org/10.1016/j.jpowsour.2016.05.051, 2016.

Worthman, E.: Recharging the Battery, Semiconductor Engineering, http://semiengineering.com/ evolving-lithium-battery-technology/ (last access: 25 April 2017), 2015.

Wu, T., Wang, M., Xiao, Q., and Wang, X.: The SOC Estimation of Power Li-Ion Battery Based on ANFIS Model, Smart Grid and Renewable Energy, 3, 51-55, 2012.

Yu, J.: State-of-Health Monitoring and Prediction of Lithium-Ion Battery Using Probabilistic Indication and State-Space Model, IEEE T. Instrum. Meas., 64, 2937-2949, 2015. 